\title{
Overview on social security system of rare diseases in China
}

\author{
Jiangjiang He ${ }^{1,2,}$, Peipei Song ${ }^{1,3,}$, Qi Kang ${ }^{1}$, Xueyan Zhang ${ }^{4}$, Jiahao Hu ${ }^{5}$, Yan Yang ${ }^{1}$, Mi Tang ${ }^{1}$, \\ Duo Chen ${ }^{1}$, Shanlian $\mathrm{Hu}^{2, *}$, Chunlin $\mathrm{Jin}^{1, *}$ \\ ${ }^{1}$ Department of Health Policy Research, Shanghai Health Development Research Center, Shanghai Medical Information Center, \\ Shanghai, China; \\ ${ }^{2}$ School of Public Health, Fudan University, Shanghai, China; \\ ${ }^{3}$ The Institute for Global Health Policy Research, National Center for Global Health and Medicine, Tokyo, Japan; \\ ${ }^{4}$ School of Economics and Management, Jiangxi University of Traditional Chinese Medicine, Nanchang, China; \\ ${ }_{5}^{5}$ Department of Learning, Informatics, Management and Ethics, Karolinska Institutet, Solna, Sweden.
}

\begin{abstract}
Summary China has initiated the special security project for rare diseases from the national strategic level since 2018. Chinese government has formulated China's First List of Rare Diseases (121 diseases included), established Expert Committee of Diagnosis, Treatment and Security of Rare Diseases and China Alliance of Rare Diseases. The government also encouraged all the provinces to include the security plan and strategies of rare diseases into key work and promoted the establishment of rare diseases clinical research center and the formulation of diagnosis and treatment guidelines. All these actions led to the further improvement of scientific research ability and diagnosis and treatment ability. In terms of the treatment drug of the 121 diseases in China's First List of Rare Diseases, 83 of them are on the market in China and 50 have been included in the national medical insurance accompanied with the tax reduction policy on rare diseases drug and several charity foundations and enterprise donation programs were developed so that drug availability and accessibility for patients are increased. However, there is no clear definition and corresponding coding of rare diseases; the economic burden of most rare diseases could not be calculated accurately because of the difficulty in diagnosis and misclassification. Regarding the service provision system, social security system of rare diseases in China needs further improvement because of the rather few kinds of rare diseases involved in the screening, low level of diagnostic ability, rather few rehabilitation programs and the lack of social caring and knowledge training. It is necessary for China to provide comprehensive and well-rounded health care and social caring for rare diseases patients. Since the formulation and implementation conditions of the policy are different among different regions, the pilot should be carried out first in regions with desired qualifications when China is formulating the rare diseases policy and then a mature national plan should be developed.
\end{abstract}

Keywords: Rare diseases, orphan drugs, social security, medical insurance, China

Released online in J-STAGE as advance publication August 20, 2019.

${ }^{\S}$ These authors contributed equally to this work.

*Address correspondence to:

Dr. Chunlin Jin, Shanghai Health Development Research Center (Shanghai Medical Information Center), Room 807, No.1477, Beijing (W) Road, Jing'an District, Shanghai 200040, China.

E-mail: jinchunlin@shdrc.org

Prof. Shanlian Hu, School of Public Health, Fudan University, No.130 Dong'an Road, Xuhui District, Shanghai 200032, China.

E-mail: hushanlian@hotmail.com

\section{Introduction}

With the new round of deepening institutional reform of healthcare in China and the medical security pilot on disastrous diseases of countryside children like leukemia and congenital heart disease (1), rare diseases are attracting more and more social concerns. Rare diseases are those with an extremely low incidence rate but there are a variety of them. They have features like complexity, severity and deterioration but are often excluded from the medical insurance system $(2,3)$. From the perspective of medicine and risk 
regulation, working on the diagnosis and treatment of rare diseases is not only an urgent need to guarantee health rights and the right to life of those patients but also an inevitable requirement for a country to realize solidarity, equity and justice, with great necessity and practical significance (4).

In recent years, China has been actively promoting the social security work of rare diseases both on the country level and the regional level. Therefore, this article plans to summarize the current status of the development of China's rare disease security system through literature review, including the definition, list and coding of rare diseases, accessibility of rare disease medication (orphan drugs), policy system of China's rare diseases, financing and payment system of rare diseases, the promotion of scientific research and standardized diagnosis and treatment of rare diseases in China and rare diseases patient organizations and service information platform. It also analyzes the problems and challenges China's rare diseases security system is facing, proposes suggestions to perfect the safeguard work and provides reference to the improvement of security and sustainability of rare diseases prevention and treatment in China.

\section{Definition, list and coding of rare diseases in China}

\subsection{Definition of rare diseases}

There is no clear official definition of rare diseases in China. On May $17^{\text {th }}, 2010$, Shanghai, Chinese Society of Genetic Medicine of Chinese Medical Association held an expert seminar about the definition of rare diseases in China. An expert consensuswas reached: Diseases with incidence rate $<1 / 500,000$ or new-born incidence rate $<1 / 10,000$ could be called a rare disease in China (5).

\subsection{List of rare diseases}

Since there are rare epidemiological data of rare diseases in China, it is a more feasible scheme to develop a rare disease list based on the real situation. The exploration of the Chinese rare disease list started from 2016. The former Shanghai Municipal Health and Family Planning Commission (now called Shanghai Municipal Health Commission) published the first provincial main list of rare diseases in 2016 - Shanghai List of Main Rare Diseases (2016 version) - which includes 56 rare diseases (6). China Organization for Rare Disorders published China's Reference List of Rare Diseases with 147 rare diseases on September 23 $3^{\text {th }}, 2016$ (7). National Health Commission of China, Ministry of Science and Technology, Ministry of Industry and Information Technology, National Medical Products Administration and National Administration of Traditional Chinese
Medicine formulated and published China's First List of Rare Diseases (hereinafter referred to as the Chinese Rare Diseases List, CRDL) on May 22 ${ }^{\text {nd }}, 2018$ (8). The main principle of this list is to put "rare diseases with a relatively higher incidence rate, heavier disease burden and better treatability" as a priority and the list includes 121 rare diseases like Gaucher disease. 50 diseases in Shanghai List of Main Rare Diseases (2016 version) and 88 in China's Reference List of Rare Diseases are involved. This list serves as a reference to each department and will help enhance the management of rare diseases in China, improve diagnosis and treatment level for rare diseases and safeguard the health interests of rare diseases patients.

On May $28^{\text {th }}, 2018$, Bureau of Medical Administration, National Health Commission of China issued Notification of Printing and Publishing the Working Procedure of the Formulation of Rare Diseases List (9), in which it was proposed that "the formulation of the list should take factors such as economic development, population and social security level into consideration based on the national condition and refer the management experience of countries or regions with similar social development level", "select the list-covered diseases in batch and update the list dynamically with a turnover time not shorter than 2 years in principle". It also points out that diseases involved in the list should meet the following requirements: there is evidence home and abroad that shows the prevalence rate or incidence rate is low; it brings great harm to patients and the family; there are definite diagnosis methods; there are treatment or intervention methods and it is economically affordable or there is no effective treatment or intervention methods but it is included in national special scientific research.

\subsection{Coding of rare diseases}

There is no official definition of rare diseases as well as individual coding system of rare diseases in China. This results in the difficulty in diagnosis and misclassification of rare diseases and makes the disease burden of most rare diseases that could not be calculated accurately. Internationally, with the approval of ICD-11 (10), there are more than 5400 kinds of rare disease that have their sole ICD-11 coding (11) and China's rare disease coding system will be perfected based on ICD-11 coding.

\section{Accessibility of rare disease medication (orphan drugs) in China}

Drug accessibility is that people could get the appropriate, highly qualified and culturally acceptable drug with an affordable price and can easily acquire information about reasonable medication, which is, accessibility, affordability and reasonable using of drugs (12). 


\subsection{Accessibility}

Among the 7000 or more known rare diseases in the whole world, only less than $10 \%$ of them have an approved treatment drug or scheme (13) and part of them are not in the China market. Taking the treatment drugs of the 121 diseases involved in CRDL as an example, 83 drugs are in the market, among which antineoplastic and immunomodulating agents, alimentary tract and metabolism and nervous system medication are the top 3 . The top 3 drugs that are involved in the national medical insurance with the most kinds are antineoplastic and immunomodulationg agents, nervous system and blood and blood forming medications (Table 1).

\subsection{Affordability}

The greatest difference between rare disease drugs and common drugs is the high price. Most rare disease patients could not afford the treatment expense with no support from a medical insurance policy. According to the report of the IQVIA institution, the median annual cost that US rare disease patients spent on single drug treatment in 2017 was 46,000 dollars (without regard to medical insurance payment). For patients of 13 rare diseases that does not have any drugs involved in medical insurance in CRDL, the median annual cost is 204,000 yuan (14). Collaborative research between School of Pharmaceutical Sciences of Peking University and China Organization for Rare Disorder (CORD) in 2013 (15) found that only less than 1.3\% of the 982 patients interviewed can completely cover the medical costs of the whole year with their annual household income. According to a survey, the annual medical cost of rare disease patients in China is 3 times the personal annual income, 1.9 times annual household income, only $17.9 \%$ of the interviewees could bear the medical cost and $76.5 \%$ mainly depend on family members (16). The phenomenon that rare disease patients' family falls into poverty or return to poverty because of illness are serious. Regarding medical insurance involvement (Table 1), by the end of 2018, 50 out of 121 rare diseases in CRDL had been involved in the national medical insurance, among which 17 are in the category A list (the drugs that are formulated uniformly by the government, must go to clinical treatment, widely used and of good efficacy with lower prices compared to drugs of the same category and are $100 \%$ reimbursed by medical insurance) and 33 are in category B (the drugs that the basic medical insurance fund will pay part of its cost. Patients need to pay the cost by themselves first and then the basic medical insurance fund will be involved with the cost and pay for it. The detailed reimbursement ratio varies according to different regional policies and the specific drug type) (17).

\subsection{Reasonable using}

China has invested many resources to support the development of the discipline of rare diseases in recent years, but the number of treatment drugs is barely enough for the great number of diseases. A survey of rare disease patients registered on the CORD (5810 patients) from 2014 to December of 2018, shows that $42 \%$ of the patients (2448) didn't receive any treatment and among the other $58 \%$ that received treatment, most could not receive timely enough drug treatment (18). Therefore, given that the accessibility of rare diseases drugs is limited, clinical doctors and researchers have to open up new approaches like "off-label" use, "old drug, new use" and "experimental treatment."

\section{Policy system of China's rare diseases}

Though China hasn't formulated a specific strategy or plan for rare diseases, no individual rare diseases commission (including civil administration, health, medical insurance, medicine administration, charity foundations, scientific research departments, etc.) has been established and patients are not involved in the decision making process in the field of rare diseases, work related to rare diseases is getting more attention, especially medication of rare diseases. "Healthy China 2030" Planning outline, a significant strategic plan, clearly points out that national medication security

Table 1. The on-market situation and medical insurance involvement of the treatment drugs for the diseases in CRDL

\begin{tabular}{|c|c|c|c|}
\hline $\begin{array}{l}\text { Anatomical therapeutic chemical classification } \\
\text { (First level) }\end{array}$ & $\begin{array}{l}\text { On the market } \\
\text { of China }(n)\end{array}$ & $\begin{array}{l}\text { Involved in the national } \\
\text { medical insurance }(n)\end{array}$ & $\begin{array}{c}\text { Ratio of medical insurance } \\
\text { involvement }(\%)\end{array}$ \\
\hline A- Alimentary tract and metabolism & 14 & 5 & 35.71 \\
\hline B- Blood and blood forming organs & 10 & 6 & 60.00 \\
\hline C-Cardiovascular system & 9 & 4 & 44.44 \\
\hline G- Genital urinary system and sex-hormones & 7 & 2 & 28.57 \\
\hline H-Systemic hormonal preparations, excluding sex hormones and insulins & 5 & 5 & 100.00 \\
\hline J-Antiinfective for systemic use & 4 & 4 & 100.00 \\
\hline L-Antineoplastic and immunomodulationg agents & 17 & 10 & 58.82 \\
\hline M-Muscular-skeletal system & 4 & 4 & 100.00 \\
\hline N-Nervous system & 13 & 10 & 76.92 \\
\hline Total & 83 & 50 & 60.24 \\
\hline
\end{tabular}


Table 2. Introduction to the policy system of rare diseases in China

\begin{tabular}{|c|c|c|}
\hline Items & Special policy theme & Comprehensive policies that mentions \\
\hline Strategy/Plan & - & "Healthy China 2030" planning outline. \\
\hline Disease & - & - \\
\hline Listing & List and its formulation procedure & - \\
\hline Study & 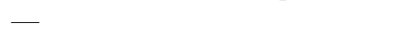 & Health scientific innovation special subject plan of "13 $3^{\text {th }}$ Five-Year Plan". \\
\hline Drug & - & "Healthy China" 2030 Planning outline. \\
\hline R\&D & - & $\begin{array}{l}\text { Guidance on promoting the healthy development of pharmaceutical } \\
\text { industry; } \\
\text { Biological industry development plan of " } 13^{\text {th }} \text { Five-Year Plan"; } \\
\text { Health plan of } 13^{\text {th }} \text { Five-Year Plan"; } \\
\text { Basic Medical and Healthcare Promotion Law of China (draft; second } \\
\text { reviewing draft). }\end{array}$ \\
\hline Marketing & - & Drug review and evaluation related policies. \\
\hline Price & VAT & - \\
\hline Medical Insurance & - & 2019 Adjustment Scheme of National Medical Insurance Drug List. \\
\hline Diagnosis and treatment service & $\begin{array}{l}\text { Diagnosis and treatment } \\
\text { collaboration network, } \\
\text { diagnosis and treatment } \\
\text { guideline }\end{array}$ & $13^{\text {th }}$ Five-Year Plan" Healthscientific innovation Subject Plan. \\
\hline
\end{tabular}

policies will be perfected (19). China has implemented a series of preferential policies for the review and approval of drugs for rare diseases, mainly including priority review and approval, accelerated review and approval, special review and approval (20). In recent years, fundamental policies about health scientific innovation and health industry development also put forward that the study of rare diseases and the development of related drug technology should be strengthened and accelerated (21-23).

The publishing of CRDL in May, 2018 (9) symbolizes that China's rare disease security work has entered into a brand-new phase. Since then, China's rare disease security has had a clear and prioritized disease scope, which laid an important foundation for the publishing of related policies (24). At the beginning of 2019, China's rare disease policies were published intensively (Table 2). Rare disease drug VAT, diagnosis and treatment collaboration network, diagnosis and treatment guidelines, etc. were covered. The adjustment scheme of the national medical insurance drug list indicated more clearly that treatment medications of serious diseases like rare diseases should have been taken into priority consideration (25). That means, a policy system of rare diseases in China is being perfected and accelerated.

\section{Financing and payment system of rare diseases in China}

\subsection{National tax reduction and medical insurance funds}

To encourage development of the rare disease pharmaceutical industry and the decrease of medication costs, State Taxation Administration has reduced the VAT of antineoplastic agents in the import process to $3 \%$ so that VAT in the domestic process could be calculated by a simplified method for the first list of 21 rare diseases drugs and 4 APIs (26). This will result in a further decline of drug costs. In addition, among all diseases in CRDL whose indications have been registered in China, some were involved in the national basic medical insurance, employment injury insurance and maternity insurance drug list or in the pilot of serious illness security $(27,28)$. Since June, 2012 , the Department of Maternal and Child Health in National Health Commission and National Maternal and Child Monitoring Office initiated the allowance program of specialized milk powder for phenylketonuria children (16).

\subsection{Medical insurance and rare diseases funds in some provinces}

Some provinces and cities in China have taken several drugs for rare diseases into local social medical insurance. There are two main security models: $i$ ) directly include the medication in the drug security list (mainly category B list or formulate for special medication list); ii) involve the disease in special disease security scope (co-paid by special financial investment and medical insurance fund) and safeguard related drugs (serious illness medical insurance, outpatient special disease in basic medical insurance or rare diseases special medical insurance). Qingdao is a classic example for the first model (such as hyperphenylalaninemia, idiopathic pulmonary arterial hypertension, Gaucher disease, etc.). It established a special drug list in 2012 through serious illness aid system (29), and transferred the list into the management of the supplementary medical insurance system in 2017 (30). Qingdao's rare diseases medical insurance has an early start, high percentage of payment, a rather complete system. Zhejiang province is typical of the second model. It initiated the rare diseases medical insurance system since 2016, which is 
recognized by basic medical insurance, serious illness medical insurance, medical aid and special aid, covering 6 rare diseases (Gaucher disease, amyotrophic lateral sclerosis, and phenylketonuria are included in the rare disease medical insurance, hyperphenylalaninemia, idiopathic pulmonary fibrosis, and idiopathic pulmonary arterial hypertension are only included in serious illness medical insurance) (31). Moreover, these two regions give admission to rare diseases drugs on the list through price negotiation and assigning designated hospitals for the diagnosis and treatment of rare diseases patients.

\subsection{Charity funds and enterprise donation programs}

Rare diseases charity funds have been continuously set up such as China-Dolls Fund for Rare Disorders and Rare Diseases Bailout Fund, which provides several forms of help to domestic rare diseases patients. Pharmaceutical companies also actively take social responsibility and initiate assistance programs with China Charity Federation. Cerezyme charity aid program supported by Sanofi-Genzyme has provided the Gaucher-disease-specific drug (Cerezyme) to more than 130 patients with severe Gaucher disease for free and the total amount of assistance until now is over 1,200 million yuan (32). The Evolocumab charity aid program supported by Amgen allows eligible patients to have Evolocumab with two years' dosage for free, which is an innovative anticholesteremic agent. There is also an hemophilia-specific Baikeqi Co-pay charity aid program and Advate charity aid program, multiplesclerosis-specific "China Charity Federation Beteferon Patients Charity Aid Program", etc. that greatly relieve the economic burden and mental stress for patients and improve their life quality (33).

\section{The promotion of scientific research and standardized diagnosis and treatment of rare diseases in China}

\subsection{Expert committee of diagnosis, treatment and security of rare diseases and rare diseases association}

In December 2015, the former National Health and Family Planning Commission (now called National Health Commission) of China formed the Expert Committee of Diagnosis, Treatment and Security of Rare Diseases whose responsibility is to study and put forward the definition and disease type that are consistent with our national circumstances, organize to develop technical specification and clinical pathways of rare diseases prevention and treatment and give suggestions for the prevention, screening, diagnosis and treatment, medication, rehabilitation, and security of rare diseases. Committee members are experts from professional fields like rare diseases screening, diagnosis and treatment, pharmacy, drug supply and security, medical insurance and health economics (34). In April, 2017, the committee office was set up in Peking Union Medical College Hospital, responsible for the daily work of the committee (35). Since 2010, Shandong, Shanghai, Beijing, Guangdong, and Zhejiang consecutively established rare diseases society or prevention and treatment association and constructed a collaborative platform of rare diseases study, diagnosis and treatment (36).

In October 2018, under the permission of Bureau of Medical Administration National Health Commission, China Alliance of Rare Diseases was set up (37). The Alliance was initiated by Peking Union Medical College Hospital, China Pharmaceutical Innovation and Research Development Association, Chinese Hospital Association and Chinese Research Hospital Association, consisting of 50 or more medical institutions and institutions of higher learning with rare disease departments, scientific research institutions and enterprises voluntarily. The Alliance is a national, nonprofit, cooperative communication platform, aiming to integrate the prevention and treatment resources of China rare diseases and motivate medical institutions, research institutions, social organizations and pharmaceutical enterprises. Tencent, China's internet giant, joined the Alliance in May, 2019 in order to apply artificial intelligence to the field of rare disease (38).

\subsection{National rare diseases scientific projects}

The pathogenesis, diagnosis and treatment methods for most rare diseases are unclear and there are few cases with patients scattered in different regions and difficulty in data collection, which makes rare diseases research still have a long way to go (39). China is gradually enhancing scientific investment by National Science and Technology Support Program, Rare Diseases Precision Medicine Research, National Major Scientific and Technological Special Project for "Significant New Drugs Development", National Natural Science Foundation of China and Special Scientific Research Fund of Public Welfare Industry of China. Since 2008, China's new drug special project has supported more than $20 \mathrm{R} \& \mathrm{D}$ projects on rare diseases. The first comprehensive rare diseases prevention and treatment research project "China's Rare Diseases Prevention and Treatment Research and Demonstration" was initiated during the period of " $12^{\text {th }}$ Five-Year Plan" and several precision medicine special research projects were set off including rare diseases clinical cohort study, precise diagnosis and treatment technology and Clinical Practice study on important rare diseases in Chinese population during the period of " $13^{\text {th }}$ FiveYear Plan" (40-42). The amount of funding has been over 120 million yuan. All these studies are based on the actual problem encountered in the field of rare diseases prevention and treatment, and the first national 
rare diseases registration system was developed (43) as well as a clinical database integrated by multiomics and multi-center clinical biobank, model rare diseases comprehensive diagnosis and treatment center and imaging expert consultation platform. Several clinical pathways were designed. All play an exemplary role in the research of rare diseases in China and the popularization and promotion of related diagnosis and treatment technologies and give strong data support for rare diseases decision-making $(36,44)$.

\subsection{Rare diseases clinical research center}

Clinical research center is a national scientific innovation base that meets the prevention and treatment needs and clinical application orientation, of which medical institutions are the main body and also a national science and technology innovation base supported by a synergetic network. It can speed up the breakthrough of the limitations of current disease diagnosis and treatment technology, explore prevention and control methods that are suitable for China and more economically efficient. The Fiveyear Development Plan for National Clinical Research Center (2017-2021) points out that 1-3 national clinical research centers will be established for birth defects and rare diseases field, focusing on structural birth defects and inborn errors of metabolism (45). After the layout of the national clinical research center for rare diseases, the ability for rare diseases screening, diagnosis and treatment in China will be further promoted.

\subsubsection{Clinical pathway}

To improve the diagnosis and treatment level of rare diseases, promote the standardization of clinical diagnosis and treatment, medical institutions in Beijing and Shanghai have been working actively on the standardization of rare diseases diagnosis and treatment. Regulations and expert consensus on the diagnosis of Gaucher disease, glycogen storage disease type II, hyperphenylalaninemia, Alport syndrome and monographs like Treatable Rare Disease and Compendium of China's First List of Rare Diseases were published in order to provide standard diagnosis and treatment guidance on some rare diseases for clinical professionals $(39,46)$. In February, 2019, Expert Committee of Diagnosis, Treatment and Security of Rare Diseases, National Health Commission (Chinese Academy of Medical Sciences \&Peking Union Medical College) led the formulation and publication of Rare Disease Diagnosis and Treatment Guide (2019) which explained in detail the definition, etiology and epidemiology, clinical feature, auxiliary examination, diagnosis, differential diagnosis and treatment and proposed detailed diagnosis and treatment processes, displaying fully that the guide is helpful in the practice of rare diseases diagnosis and treatment in terms of standardization, guidance and practicality (47).

\subsubsection{Diagnosis}

The diagnosis and treatment service system of rare diseases in China is still being constructed preliminarily. There is difficulty in diagnosis and misdiagnosis and missed diagnosis. The average length of definite diagnosis is 5-6 years and it is more than 10 years for some patients (48). In February, 2019, National Health Commission of China published National Health Commission General Office's Notification on the establishment of national rare diseases diagnosis and treatment collaborative network (Medical Letter of National Health Commission General Office (2019) No.157), and decided to select 324 hospitals in the whole country with stronger diagnosis and treatment capabilities for rare diseases and more cases to form the rare diseases diagnosis and treatment collaborative network. This helps to realize the objective of early detection, early diagnosis, treatability and manageability of rare diseases gradually (49). 80\% of rare diseases are caused by genetic variation and with development of molecular diagnosis, molecular genetics, and gene sequencing technology (50), diagnosis rate of rare diseases increases significantly, length of definite diagnosis shortens and cost declines. In addition, some regions in China are able to choose to use tandem mass spectrometry for the detection of each rare disease $(51) .13^{\text {th }}$ Five-Year Plan for Development of the Strategic Emerging Industries also points out that high performance medical devices and core components should be developed to support the in vitro rapid and accurate diagnosis and screening of cancer, hereditary diseases and rare diseases (52).

\subsubsection{Screening}

Most of the rare diseases are caused by genetic variation, so new-born disease screening could effectively recognize rare diseases that have not emerged. China's new-born diseases screening began from the 1980s. With the publishing of related policies, laws and supporting documents, the preconception and antenatal examination and new-born screening system are continuously improving. Screening has developed quickly with more screening types and a nationwide new-born screening network has developed with a new-born screening coverage rate of $97.5 \%$ (53). Diseases involved in the nationwide screening are new-born inherited metabolic disorders like congenital hypothyroidism, phenylketonuria and hearing disorders (54). What's more, two experts in the field of rare diseases screening from Expert Committee of Diagnosis, Treatment and Security of Rare Diseases are assigned to offer suggestions (55). 


\subsubsection{Rehabilitation}

Most rare diseases are chronic and serious disease due to gene defects, with a few complications and cannot be cured thoroughly, causing many patients to receive treatment or rehabilitation for their whole life (56). According to a survey, the greatest difficulty rare diseases patients meet in the treatment process is over the high treatment expense, followed by the lack of drugs and rehabilitation methods (57) that seriously influence life quality. Professional rehabilitation therapists are needed to comprehensively evaluate the physical and psychological status of patients and formulate an individualized rehabilitation scheme. Some rare diseases organizations have initiated programs like rehabilitation training camp/ rehabilitation talents development projects (58) and Expert Committee of Diagnosis, Treatment and Security of Rare Diseases should also fully play their role (55). Moreover, rare diseases patients are restricted in education or employment, and often isolated and discriminated against in daily life. These bring great psychological pressure to them (59) while their family is heavily burdened. Apart from the support of advanced medical technology and finance, patients need certain social support and mental rehabilitation. In China, there are only a few NGOs that could offer help for education, employment and mental health and most can only provide some medical aid or life help services for now (58).

\section{Rare disease patient organizations and service information platform}

\subsection{Rare diseases patient organizations}

Chinese hemophiliacs developed a patient communication and mutual help platform since the beginning of the $21^{\text {st }}$ century. Since then, rare diseases patients organizations in China began to emerge and develop (60). The Chongqing Hemophilia Rehabilitation Association was set up in 2006 and is the first rare diseases patients organization registered in China civil administrations (61). There are currently about 80 registered rare diseases patients' organizations (62). Besides these patient organization for specialized diseases, another patient platform organization (CORD) was established in 2013 and played an important role in promoting the development of Chinese rare diseases patient organizations, including the ability training of patient organizations, activity support and social publicity (63). Meanwhile CORD also conducts policy research, patient registration and international collaboration.

Because of the particularity of rare diseases, misdiagnosis or missed diagnoses often happen because of clinical physicians' insufficient understanding. It is of great significance to have a wide-range of rare diseases prevention and control publicity and training of specialist physicians in order to standardize diagnosis and treatment, relieve patients' pain and avoid the waste of medical resources (64). National and local health commissions, together with medical institutions, carry out some knowledge training about rare diseases and rare disease organizations also regularly hold ability cultivation training. But the trainings should be enhanced, and a training system still needs to be developed.

\subsection{Rare diseases service information platform}

The main information source for rare disease patients are patient organizations established spontaneously (CORD, Chinese Organization for Albinism, ChinaDolls Center for Rare Disorders, etc.), websites (Rare Disease in China, National Rare Diseases Registry System of China, Shandong Rare Diseases Prevention and Control Association, etc.), exchange meetings (China Rare Diseases Summit, Cross-strait exchange forum of rare diseases, Asian rare diseases patients exchange forum, etc.), remote consultation platform of rare diseases/difficult diseases, patient groups, and related books and reports. Patients could acquire rare diseases related policies, scientific and technological knowledge, orphan drug information, activities home and abroad and could get access to rare diseases consulting websites from other countries or regions like EU.

\section{Conclusion}

Rare disease is a new policy hotspot in the current policy environment of China and the policy of rare disease security system construction is in its window phase. The central government's effort promotes each province to involve the security plan and strategy as the key emphasis in work and also promotes the establishment of rare disease clinical centers and the formulation of diagnosis and treatment guidelines. The Rare diseases security system is developed and implemented on the country level and consists of a series of comprehensive and all-round rare diseases related health policies and social policies. The reasonable allocation of human resources, financial resources and infrastructures plays an important role in the development of the national rare diseases security system. The experience of EU shows that (65), the formulation of rare diseases security system should put emphasis on the following two aspects: i) Comprehensiveness. Related strategies could complement each other to the greatest extent and not overlap; ii) All-roundness. Most rare diseases patients' needs should be fully considered (such as medical services and social services). It is necessary for China to formulate a specific national plan and strategy for rare diseases, provide comprehensive and all-round 
health care and social caring for rare disease patients and strengthen the collaboration between regions home and abroad. Since the formulation and implementation conditions of the policy are different among each region, the pilot should be carried out first in regions with desired qualifications when China is formulating the rare disease policy and then a mature national plan should be developed. This is to ensure the accessibility and equity of different regions regarding the diagnosis and treatment services and drugs for rare diseases.

\section{Acknowledgements}

This study was supported by grants from the Shanghai Municipal Health Commission for a Special Research Project related to Health Policies entitled "Study on the disease burden of and social security for the vulnerable population in Shanghai (Project number: 19Y04017)" and China Medical Board Collaborating Project "Establishing Health Policy Transformation Network of China (Project number: CMB-CP 14-190)".

\section{References}

1. National Health Commissioin of the People's Republic of China. Opinions on Piloting Work to Improve the Level of Medical Insurance for Major Diseases in Rural Children. http://www.gov.cn/ztzl/ygzt/content_1661153. htm (accessed May 25, 2019). (in Chinese)

2. European commission. Communication from the Commission to the European Parliament, the Council, the European Economic and Social Committee and the Committee of the Regions on Rare Diseases: Europe's challenges. Brussels, 11.11.2008 COM (2008). http:// ec.europa.eu/health/ph_threats/non_com/docs/rare_ com_en.pdf (accessed May 25, 2019).

3. European commission. Council Recommendation of 8 June 2009 on an action in the field of rare diseases OJ (2009/C 151/02). http://eur-lex.europa.eu/LexUriServ/ LexUriServ.do? uri=OJ:C:2009:151:0007:0010:EN:P DF (accessed May 25, 2019).

4. Cass R. Sunstein. Risk and reason: Safety, law, and environment (Shi S, Translate). University of Political Science and Law Press. Beijing, China, 2005; pp.165.

5. Ma D, Li DG, Zhang X, He L. Opportunities and challenges in the prevention and treatment of rare diseases in China. Chinese Journal of Evidence-based Pediatrics. 2011; 6:81-82. (in Chinese)

6. Shanghai Municipal Government. Notice of the Municipal Health and Family Planning Commission on Printing and Distributing the List of Major Rare Diseases in Shanghai (2016 Edition). http://www.shanghai.gov. cn/nw2/nw2314/nw2319/nw12344/u26aw46702.html (accessed May 25, 2019) (in Chinese)

7. China Organization for Rare Disorders. The 7th China Rare Disease Summit was opened in Hangzhou in 2016. http://www.cord.org.cn/news/211.html (accessed May 26, 2019). (in Chinese)

8. Wu JN. List promotes the leap-forward development of rare diseases. China Hospital CEO. 2018; 12:24-25. (in Chinese)
9. National Health Commissioin of the People's Republic of China. Notification of Printing and Publishing the Working Procedure of the Formulation of Rare Diseases List. http://www.nhc.gov.cn/yzygj/s7659/201806/927def ed71934ec8b032ccfca0afe0fb.shtml (accessed May 26, 2019). (in Chinese)

10. World Health Organization, WHO releases new International Classification of Diseases (ICD 11). https:// www.who.int/zh/news-room/detail/18-06-2018-whoreleases-new-international-classification-of-diseases(icd-11). (accessed May 27, 2019).

11. SégolèneAymé, Bertrand Bellet, Ana Rath. Rare diseases in ICD11: Making rare diseases visible in health information systems through appropriate coding. Orphanet J Rare Dis.2015; 10:35.

12. Ying L, Yi L. Accessibility and patent protection of drugs. China Pharmacy. 2007; 18:966-968. (in Chinese)

13. Ge L, Wei CJ, Shi LW, Ding J. Study on current medication situation of rare disease in China. Beijing Medical Journal. 2018; 40:432-434. (in Chinese)

14. Rare disease information network. The China Rare Disease Drug Accessibility Report (2019) is solemnly released in Beijing. http://www.hanjianbing.org/sear ch!detail id $=8 a 9 d 475463869 c 1901693940728 b 04 b 2$ (accessed April 21, 2019). (in Chinese)

15. Fu MY, Guan XD, Wei GX, Xin XX, Shi LW. Medical service utilisation, economic burden and health status of patients with rare diseases in China. Journal of Chinese Pharmaceutical Sciences. 2018; 27:361-369. (in Chinese)

16. Ding J, Wang L. Report of rare diseases in China (2018) (Ding J, Wang L, eds). China Medical Science Press, Beijing, China, 2018; pp. 12-36. (in Chinese).

17. $\mathrm{Xu} \mathrm{W}$. The payment standard of medical insurance should be a dynamic standard. China Health Insurance.2016; 7:21-22. (in Chinese)

18. Shao WB, Li YY, Wang F, Zhu YJ, Xiao L, Huang RF. Accessibility of rare drugs in China and Solutions. China Food \& Drug Administration Magazine. 2019; 2:8-15. (in Chinese)

19. Central People's Government of the People's Republic of China. "Healthy China 2030" Planning Outline" issued by the Central Committee of the Communist Party of China and the State Council. http://www.gov. cn/zhengce/2016-10/25/content_5124174.htm (accessed May 15, 2019). (in Chinese)

20. Yang Y, Kang Q, Hu JH, Kong FX, Tang M, He JJ, Jin CL. Accessibility of drugs for rare diseases in China: Policies and current situation. Intractable Rare Dis Res. 2019; 8:80-88.

21. National Development and Refrom Commission of China. Guiding Opinions of the General Office of the State Council on Promoting the Sound Development of the Medical Industry. http://www.ndrc.gov.cn/fzgggz/ gyfz/gyfz/201603/t20160317_793888.html (accessed June 5, 2019). (in Chinese)

22. National Development and Reform Commission of China. Outline of the 13 th Five-Year Plan for the Biological Industry. http://www.ndrc.gov.cn/fzgggz/ fzgh/ghwb/gjjgh/201706/t20170605_849990.html (accessed June 5, 2019). (in Chinese)

23. Central People's Government of the People's Republic of China. The 13th Five-Year Plan for Healthcare issued by the State Council. http://www.gov.cn/xinwen/2017-01/10/ content_5158559.htm (accessed June 8, 2019). (in Chinese) 
24. He JJ, Kang Q, Hu JH, Song PP, Jin CL. China has officially released its first national list of rare diseases. Intractable Rare Dis Res. 2018; 7:145-147.

25. Central People's Government of the People's Republic of China The Work Plan of National Basic Medical Care Insurance Medicine Catalogue Adjustment issued by the National Healthcare Security Administration. http:// www.gov.cn/xinwen/2019-04/19/content 5384349.htm (accessed June 10, 2019). (in Chinese)

26. Ministry of Finance of China. Notice on value-added tax policy for rare diseases. http://szs.mof.gov.cn/ zhengwuxinxi/zhengcefabu/201902/t20190222_3176415. html (accessed July 10, 2019) (in Chinese)

27. Ministry of Finance of China. Notice on Doing a Good Job of New Rural Cooperative Medical Care in 2012. http://szs.mof.gov.cn/mofhome/shehuibaozhangsi/ zhengwuxinxi/zhengcefabu/201205/t20120530 655597. html (accessed July 10, 2019) (in Chinese)

28. Pan F. Establish a long-term mechanism to ensure the safety of medication for special populations. China Medical Herald. 2019; 16:6-8. (in Chinese)

29. Qingdao Municipal Government. Notice on Forwarding the Opinions of the Municipal Human Resources and Social Security Bureau and Other Departments on Establishing a Medical Assistance System for Urban Severe Diseases (Trial). http://www.qingdao.gov.cn/ $n 172 / n 68422 / n 1527 / n 27217087 / 121018100219573382$. html (accessed July 5, 2019). (in Chinese)

30. Qingdao Municipal Government. Implementation Comments on the Establishment of a Supplementary Medical Insurance System. http://www.qingdao.gov.cn/ n172/n68422/n1527/n31280741/170104160643494113. html (accessed July 5, 2019). (in Chinese)

31. ZheJiang Civil Affairs Bureau. Notice on Strengthening Medical Care for Rare Diseases issued by ZheJiang Human Resources and Social Security Bureau, ZheJiang Civil Affairs Burea, ZheJiang Finance Bureau and ZheJiang Health and Family Planning Commission. http://www.zjmz.gov.cn/il.htm? $a=$ si\&id=8aaf8015571 7920d0157417ef0e202a9 (accessed July 5, 2019). (in Chinese)

32. Jin M, Ma AX. Research on the Policies of Rare Diseases and Orphan Drug Supply Security Modes in Jiangsu Province. China Journal of Pharmaceutical Economics.2019; 14:93-101. (in Chinese)

33. China Charity Federation. Charity project. http:// www.chinacharityfederation.org/ProjectList/29/3.html (accessed July 10, 2019) (in Chinese)

34. National Health Commissioin of the People's Republic of China. Notice of the General Office of the former National Health and Family Planning Commission on the Establishment of the National Health and Family Planning Commission Expert Committee on the Diagnosis and Treatment of Rare Diseases. http://www. nhc.gov.cn/yzygj/s7659/201601/c918591a06154c1ea 193fc32ba93c085.shtml (accessed July 1, 2019). (in Chinese)

35. National Health Commissioin of the People's Republic of China. Notice of the General Office of the former National Health and Family Planning Commission on the addition and adjustment of some members of the Expert Committee on the Diagnosis and Treatment of Rare Diseases. https://dwz.cn/7sxRWeZp (accessed July 6, 2019). (in Chinese)

36. Xu HP, Zhu C, Gong MC, Zhang SY. Research of Rare
Diseases in China: From the Past to the Future. Medical Journal of Peking Union Medical College Hospital. 2018; 9:5-9. (in Chinese)

37. ScienceNet. China Rare Disease Alliance was established in Beijing. http://news.sciencenet.cn/ htmlnews/2018/10/419099.shtm (accessed July 6, 2019). (in Chinese).

38. CN-Healthcare. Tencent has joined Chian Rare Disease Alliance. https://www.cn-healthcare.com/ article/20190526/content-519523.html (accessed July 6, 2019). (in Chinese)

39. Zang SY. Diagnosis and treatment of rare diseases in China. Clinical Focus. 2019; 34:197-200. (in Chinese)

40. He JJ, Tang M, Zhang XY, Chen D, Kang Q, Yang Y, $\mathrm{Hu} \mathrm{JH}$, Jin $\mathrm{CL}$, Song PP. Incidence and prevalence of 121 rare diseases in China: Current status and challenges. Intractable Rare Dis Res. 2019; 8:89-97.

41. Development Center for Medical Science \& Technology, National Health Commission of the People's Republic of China. Notice on publicizing the 2016 annual project arrangement of the National Key Research and Technology Development Program "Precision Medical Research". http://www.dcmst.org.cn/tz3/287-2016111 ? from $=$ groupmessage $\&$ isappinstalled $=0$ (accessed July 10, 2019) (in Chinese)

42. National Science and Technology Management Information System and Public Service Platform. Notice on the Publicity of the National Key Research and Development Program "Precision Medical Research" and "Reproductive Health and Major Birth Defect Prevention and Control Research". http://service.most. gov.cn/2015tztg all/20170602/2189.html (accessed July 10, 2019) (in Chinese)

43. Song PP, He JJ, Li F, Jin CL. Innovative measures to combat rare diseases in China: The national rare diseases registry system, larger-scale clinical cohort studies, and studies in combination with precision medicine research. Intractable Rare Dis Res. 2017; 6:1-5.

44. Ministry of Science and Technology. The "Twelfth Five-Year" National Science and Technology Support Program "China Rare Disease Prevention Research and Demonstration" project passed the acceptance. http:// www.most.gov.cn/kjbgz/201607/t20160726_126785.htm (accessed July 10, 2019) (in Chinese)

45. Ministry of Science and Technology of China. Notice on Printing and Distributing three Documents of the National Clinical Medical Research Center Five Years (2017-2021) Development Plan. http://www.most.gov. cn/mostinfo/xinxifenlei/fgzc/gfxwj/gfxwj2017/201709/ t20170907 134799.htm (accessed July 10, 2019) (in Chinese)

46. Luo XP, Lang Y. Present situation of inherited metabolic diseases in children in China and the counter measures. Chinese Journal of Practical Pediatrics. 2014; 29:561564. (in Chinese)

47. Pan F. Constructing a research service system for the diagnosis and treatment of rare diseases in China Interview with Prof. Zhang Yuyang, Vice President of Peking Union Medical College Hospital. China Medical Herald. 2019; 16:1-3. (in Chinese)

48. Zhang SY. Caring for rare diseases, starting with the use of drugs. China Health. 2019; 5:64-65.

49. National Health Commissioin of the People's Republic of China. Notice on the establishment of a national network for the diagnosis and treatment of rare diseases. http:// 
www.nhc.gov.cn/yzygj/s7659/201902/3a8228589bf94e6 d9356008763387cc4.shtml (accessed July 10, 2019) (in Chinese)

50. Wang W. Progress in diagnosis and treatment of rare diseases from the perspective of heredity. Clinical Focus. 2019; 34:201-206. (in Chinese)

51. Kang Q, Hu JH, Song PP, He JJ. System building and improvement for the diagnosis and treatment of rare diseases in Shanghai, China. Intractable Rare Dis Res. 2018; 7:291-294.

52. The Central People's Government of the People's Republic of China. Notice of the State Council on Printing and Distributing the National 13th Five-Year National Strategic Emerging Industry Development Plan. http://www.gov.cn/zhengce/content/2016-12/19/ content_5150090.htm (accessed July 10, 2019) (in Chinese)

53. Zhao ZY. Screening progress of newborns inherited metabolic diseases. Chinese Journal of practical Pediatrics. 2014; 29:586-589. (in Chinese)

54. National Health Commission of the People's Republic of China. Notice of the General Office of the Ministry of Health on Printing and Distributing the National Plan for Screening Neonatal Diseases. http://www.nhc.gov.cn/ wjw/gfxwj/201304/47d98724198940fe81d0861e9a07cc 5a.shtml (accessed July 10, 2019) (in Chinese)

55. National Health Commission of the People's Republic of China. Interpretation of the Notice on Establishing the Expert Committee of National Health and Family Planning Commission for the Diagnosis and Treatment of Rare Diseases. http://www.nhc.gov.cn/yzygj/s7659/2016 01/04074bc9f66943e09f2dafd04ffa5583.shtml (accessed July 10, 2019) (in Chinese)

56. Ji WX, Fan B. Analysis on Family Pressure of Families with Rare Disease Children - Based on Ecological System Process Integration Perspective. Disability Research. 2015; 1:24-30. (in Chinese)

57. Ma Z, Zhen XY. Analysis of health service utilization among patients with rare diseases and the influencing factors in China. Chinese Journal of Health Policy. 2018; 11:56-60. (in Chinese)

58. Liang SK. Social work services for patients with rare diseases: Real needs, main difficulties and countermeasures. Social Work and Management. 2016; 16:13-22. (in Chinese)

59. Biao W, Xiao YK, Chen R, Qin X, Ma Y, Hu Z. Study on social support of rare disease sufferers in China. Medicine and Society. 2012; 25:53-55. (in Chinese)

60. Huang RF, Wei YS, Hu JH, Kong FX, He JJ, Yang Y, Tang M, Jin CL, Kang Q . The progress of, challenges faced by, and future of rare disease patient organizations in China. Intractable Rare Dis Res. 2019; 8:158-160.

61. Xiao L. Roles of patient organizations in rare disease research and orphan drug development. Journal of International Pharmaceutical Research. 2017; 44:209214. (in Chinese)

62. Sohu net. China's first large-scale platform for communication among organizations of patients with different diseases has been established. http://www.sohu. com/a/282884350_100116018 (accessed July 5, 2019). (in Chinese)

63. China Organization for Rare Disorders. Brief Introduction. http://www.cord.org.cn/nav/2.html (accessed June 25, 2019). (in Chinese)

64. Sohu Net. Strengthening the training of rare diseases prevention and treatment - the National Health and Health Commission's rare disease experts walked into Hebei. http://www.sohu.com/a/256268086_434123 (accessed July 5, 2019). (in Chinese)

65. He JJ, Zhang YB, Xia SJ, Hu SL. Social security systemof rare diseases in European Union and its implications for China. Chinese Journal of Health Policy. 2012; 5:52-59. (in Chinese)

(Received June 12, 2019; Revised August 6, 2019; Accepted August 12, 2019) 